\title{
Processo de validação de instrumentos de autoavaliação da voz no Brasil
}

\author{
Validation process of voice self-assessment instruments in Brazil
}

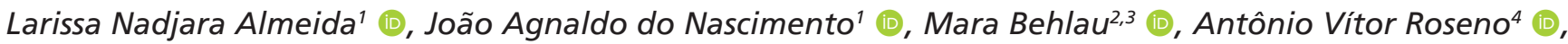 \\ Alexandra Aguiar ${ }^{1}$ (D), Anna Alice Almeida ${ }^{1}$ (i)
}

\section{RESUMO}

Objetivo: caracterizar e refletir sobre o processo de equivalência cultural e/ou validação de instrumentos de autoavaliação vocal traduzidos e adaptados para o português brasileiro, utilizados na prática clínica e em pesquisas científicas. Estratégia de pesquisa: Buscas realizadas nas bases de dados: SciELO, LILACS, PubMed e The Cochrane Library. Critérios de seleção: estudos selecionados quanto à presença dos descritores citados em seu título, resumo ou lista de descritores; artigos de validação de instrumento de autoavaliação em voz, ou com o objetivo de realizar equivalência cultural; direcionados à população brasileira; estudos originais com amostra de seres humanos, independentemente da idade do ciclo vital, tipo de disfonia ou sintomas vocais. Resultados: nove artigos selecionados. Qualidade de vida em voz e desvantagem vocal foram os construtos mais abordados. A maioria das validações ocorreu na Região Sudeste do Brasil. A maior parte dos escores dos instrumentos foi calculada por somatório simples das respostas dos participantes nos itens; pontos de corte nem sempre foram apresentados. Os domínios dos instrumentos, bem como os itens que os compunham, foram mantidos conforme apresentado no instrumento em sua língua original. A estatística mais comum para análise dos instrumentos foi o coeficiente Alfa de Crombach. Conclusão: os construtos mais abordados nos artigos selecionados foram qualidade de vida em voz e índice de desvantagem vocal. Os instrumentos foram considerados válidos e sensíveis para autoavaliação vocal, mesmo quando não consideradas as etapas propostas internacionalmente para validação de instrumentos em saúde.

Palavras-chave: Estudos de validação; Inventários e questionários; Autoavaliação; Disfonia; Distúrbios da voz

\begin{abstract}
Purpose: to characterize and reflect on the process of cultural equivalence and/or validation of vocal self-assessment instruments translated and adapted to the Brazilian Portuguese, used in clinical practice and scientific research. Research strategy: The search of articles was carried out in the following databases: SciELO, LILACS, PubMed and "The Cochrane Library". Selection criteria: The studies were selected regarding the presence of the descriptors cited in their title, abstract or in the list of descriptors; validation articles of a self-assessment instrument in voice or with the objective of performing cultural equivalence; to be directed to the Brazilian population; original studies with a sample of human beings; regardless of the age of the life cycle, type of dysphonia or vocal symptoms. Results: Nine articles were selected. Quality of life in voice and vocal handicap were the most addressed constructs. Most validations occurred in the Southeast region of Brazil. The scores of the instruments is calculated by a simple sum of the answers given by the participants in the items; cutoff points are not always presented. The domains of the instruments, as well as the items that compose them, were maintained as presented in the instrument in its original. The most common statistic for instrument analysis was crombach's Alpha coefficient. Conclusion: The constructs most addressed in the selected articles were quality of life in voice and vocal handicap index. The instruments were considered valid and sensitive for vocal self-assessment, even not considering the international proposals for validation of health instruments.
\end{abstract}

Keywords: Validation Studies; Inventories and Questionnaires; Self-assessment; Dysphonia; Voice Disorders

\footnotetext{
Trabalho realizado no Programa de Pós-graduação em Modelos de Decisão e Saúde, Universidade Federal da Paraíba - UFPB - João Pessoa (PB), Brasil.

${ }^{1}$ Programa de Pós-graduação em Modelos de Decisão e Saúde da Universidade Federal da Paraíba - UFPB - João Pessoa (PB), Brasil.

${ }^{2}$ Universidade Federal de São Paulo - UNIFESP - São Paulo (SP), Brasil.

${ }^{3}$ Centro de Estudos da Voz - CEV - São Paulo (SP), Brasil.

${ }^{4}$ Centro Universitário de João Pessoa - UNIPÊ - João Pessoa (PB), Brasil.

Conflito de interesses: Não.

Contribuição dos autores: $L N A$ responsável pela concepção e delineamento do estudo, coleta e análise dos artigos selecionados e redação do artigo; JAN responsável pela redação e por revisar o artigo de forma intelectualmente importante; $M B$ responsável pela redação e revisão do artigo de forma intelectualmente importante e aprovação final da versão a ser publicada; $A V R$ responsável pela coleta e análise dos artigos selecionados e redação do artigo; $A A$ responsável pela coleta e análise dos artigos selecionados e redação do artigo; $A A A$ contribuiu na orientação, redação e revisão do artigo de forma intelectualmente importante e aprovação final da versão a ser publicada.
}

Financiamento: Este estudo faz parte de um projeto de pesquisa financiado pelo Conselho Nacional de Desenvolvimento Científico e Tecnológico (CNPq), no processo ${ }^{\circ} 303503 / 2016-5$.

Autor correspondente: Anna Alice Almeida. E-mail: anna_alice@uol.com.br

Recebido: Julho 17, 2020; Aceito: Novembro 26, 2020 


\section{INTRODUÇÃO}

A avaliação vocal deve ser interdisciplinar, multidimensional e holística ${ }^{(1-3)}$, já que a produção da voz é um fenômeno complexo e envolve questões biopsicossociais ${ }^{(1,2)}$. Dessa forma, qualquer mensuração vocal que utilize apenas um parâmetro pode ser considerada, no mínimo, reducionista ${ }^{(4)}$, visto que estudos ${ }^{(4-7)}$ sugerem a inclusão de aspectos, como exame laríngeo, análises perceptivoauditiva da qualidade vocal e acústica de diversos parâmetros componentes do som, aerodinâmica da voz, exame corporal básico, avaliação da expressividade, levantamento da exposição a fatores de risco e autoavaliação do impacto do problema de voz, na perspectiva do paciente ${ }^{(5-7)}$.

A perspectiva do indivíduo tem sido valorizada nas últimas três décadas e compõe o conceito de saúde da Organização Mundial de Saúde-OMS ${ }^{(8)}$, cujo posicionamento causou grande impacto na área de voz clínica, de tal forma que, atualmente, não se considera completa uma avaliação vocal que não inclua a perspectiva do paciente sobre sua voz e sobre os prejuízos relacionados ao problema de $\mathrm{voz}^{(8-12)}$.

A autoavaliação de qualquer aspecto relacionado à voz contribui para a reflexão sobre o fenômeno vocal, aumenta a percepção do problema e permite a melhor compreensão sobre a comunicação em geral e comportamento vocal do indivíduo, o que, muitas vezes, não é observável e nem relatado espontaneamente no ambiente clínico, além de trazer informações que não são obtidas por nenhuma outra dimensão avaliativa ${ }^{(9-11)}$. A correlação entre a avaliação fonoaudiológica clínica e a autoavaliação do paciente, embora seja positiva, é de força muito fraca ${ }^{(12,13)}$, não permitindo a inferência da percepção do paciente.

Há vários instrumentos ${ }^{(14-18)}$ e construtos utilizados para verificar a autoavaliação da pessoa que apresenta queixas/ problemas vocais, que têm se tornado mais frequentes na clínica e em pesquisas. Esses instrumentos e construtos abordam aspectos como comprometimento da qualidade de vida em voz, sintomas relacionados a problemas na laringe e/ou na voz, percepção de desvantagem devido a alguma alteração vocal e tipo de enfrentamento na disfonia, adesão à terapia, entre outros $^{(7,9-11)}$. Geralmente, são apresentados como questionários com perguntas fechadas, que representam uma estratégia rápida, não invasiva e de fácil manejo para obtenção de informações imprescindíveis na decisão do diagnóstico vocal ${ }^{(19,20)}$. Muitos permitem a quantificação do impacto causado pela disfonia, avaliação da evolução do paciente e respaldo de decisões terapêuticas ${ }^{(11,19,20)}$.

Esses protocolos, de maneira geral, foram construídos e validados no inglês americano ${ }^{(14-18)}$ e se configuram como instrumentos de medida, compostos por um conjunto de itens, cujas respostas são, comumente, categorias ordenadas em escala Likert $^{(21)}$, uma estratégia psicométrica amplamente utilizada, que permite conhecer o nível de concordância do respondente, além de escalas nominais, dicotômicas ou ordinais, intervalares e proporcionais. Essas chaves de respostas são utilizadas no cálculo de escores dos questionários, capazes de estimar as características/variáveis relacionadas ao aspecto investigado, também chamado de traço latente ${ }^{(22)}$.

Pesquisadores brasileiros rapidamente ${ }^{(9,14-18)}$ compreenderam a relevância do uso de tais instrumentos e se comprometeram em adaptar culturalmente, traduzir e validar para o português brasileiro alguns desses questionários que se propõem mensurar aspectos vocais, para contribuir na determinação da presença de um problema de voz.

Esses processos de validação frequentemente têm como base as etapas do Scientific Advisory Committee (SAC), do Medical Outcomes Trust ${ }^{(23)}$, que são as mais utilizadas em saúde e no Brasil, sendo elas: modelo conceitual e de medida; confiabilidade; validade; sensibilidade; interpretabilidade; demanda de administração e resposta; modos alternativos de aplicação e adaptação linguística e cultural.

Dessa forma, com o crescimento dos estudos de validação de instrumentos de autoavaliação vocal no Brasil, sua aplicação e utilização na prática clínica e em pesquisas científicas, é importante observar e descrever seus processos metodológicos e etapas seguidas. Sendo assim, buscou-se responder à seguinte pergunta norteadora: "Como é realizado o processo de validação para o português brasileiro dos instrumentos de autoavaliação vocal propostos na literatura, em relação ao seu desenvolvimento, análise e propriedades psicométricas?"

\section{OBJETIVO}

O objetivo desta revisão sistemática foi caracterizar e refletir sobre o processo de equivalência cultural e/ou validação de instrumentos de autoavaliação vocal traduzidos e adaptados para o português brasileiro, utilizados na prática clínica e em pesquisas científicas.

\section{ESTRATÉGIA DE PESQUISA}

Trata-se de uma revisão sistemática da literatura, que abordou a validação de instrumentos de autoavaliação vocal e foi realizada por meio da busca de artigos relacionados ao tema nas bases de dados SciELO, LILACS, PubMed e The Cochrane Library, desenvolvida em novembro de 2019, seguindo a recomendação PRISMA (Reporting Items for Systematic Reviews and Meta-Analyses) $)^{(24)}$.

Utilizaram-se para a busca os Descritores em Ciências da Saúde (DeCS), em português, e seus correspondentes em inglês: Distúrbios da Voz/Voice Disorders e Disfonia/Dysphonia, associados ao operador booleano $A N D$, a Estudos de Validação/ Validation Studies e Inquéritos e Questionários/Surveys and Questionnaires. Desse modo, formaram-se as seguintes combinações: Voice Disorders and Validation Studies, Voice Disorders and Surveys and Questionnaires, Dysphonia and Validation Studies e Dysphonia and Surveys and Questionnaires.

\section{CRITÉRIOS DE SELEÇÃo}

Foi realizada a leitura de títulos, resumos e artigos completos, selecionados conforme os seguintes critérios de elegibilidade: descritores citados em seus títulos, resumos ou palavras-chave; artigos de validação de questionário de autoavaliação em voz ou com objetivo de realizar equivalência cultural; direcionados à população brasileira; estudos originais com amostra de seres humanos; d) abranger qualquer idade do ciclo vital e tipo de disfonia ou sintomas de problemas vocais.

A pesquisa não foi restritiva quanto à língua e ao ano de publicação. Os artigos presentes em mais de uma base de 
dados e/ou pesquisa por palavras- chave foram contabilizados apenas uma vez. O método de seleção dos artigos está descrito na Figura 1.

\section{ANÁLISE DOS DADOS}

A pesquisa nas bases de dados foi realizada por dois revisores, de forma independente, e ocorreu, inicialmente, a partir da leitura e avaliação dos títulos e resumos dos artigos encontrados, de acordo com os descritores, observando-se a relação com o tema proposto e enquadramento nos critérios de seleção. Em seguida, os estudos foram encaminhados para avaliação do texto completo. As divergências entre os avaliadores foram reanalisadas por um terceiro avaliador, a fim de saná-las e, assim, incluir ou excluir o artigo em questão. Os manuscritos que atenderam aos critérios de elegibilidade foram selecionados para análise e categorização dos dados, a partir dos seguintes aspectos: objetivos; ano de publicação; local de validação; nome do instrumento; construto; população-alvo (patologia, sexo e idade); tamanho da amostra; obtenção dos escores; testes estatísticos; se houve aplicação pré, durante e pós-terapia; conclusões.

Os dados foram apresentados sistematicamente, seguindo a ordem cronológica de publicação do artigo e com destaque aos aspectos preestabelecidos.

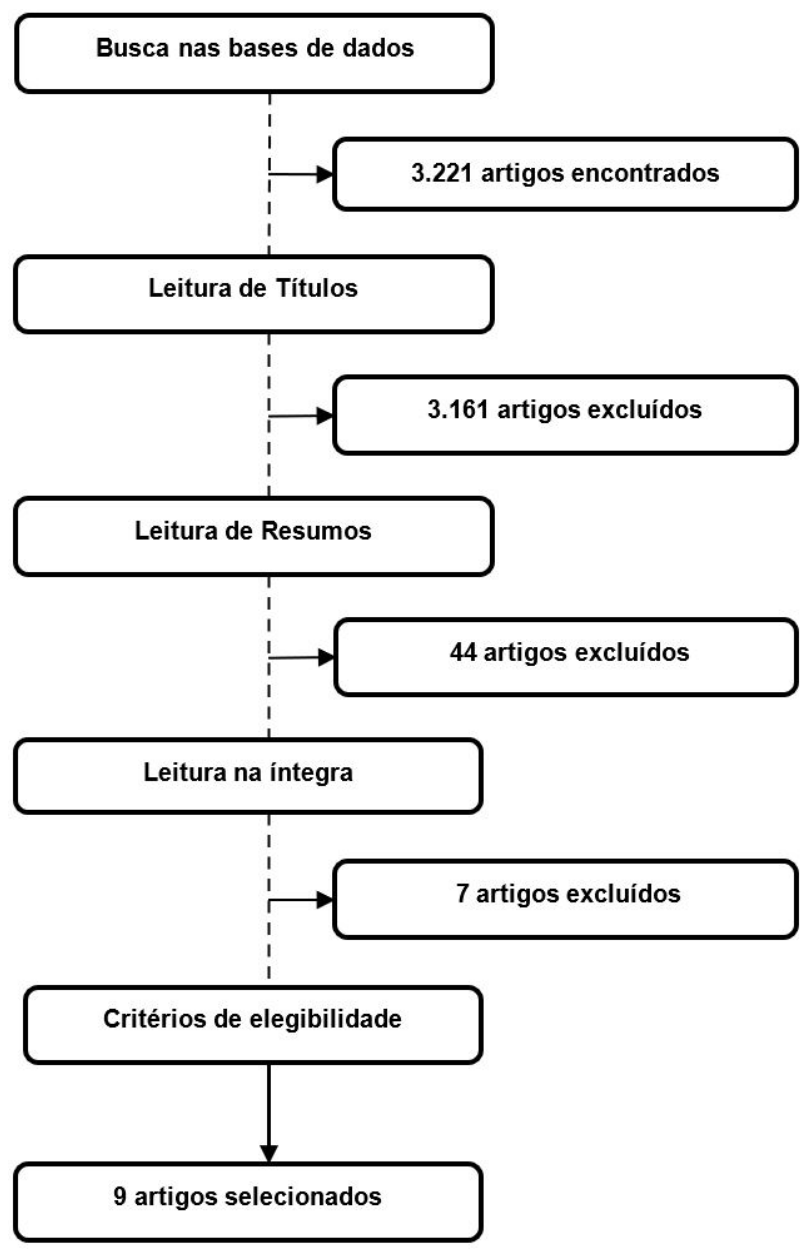

Figura 1. Estratégias para seleção dos artigos para revisão sistemática

\section{RESULTADOS}

Foram encontrados 3.221 artigos nas bases de dados preestabelecidas. Após a leitura dos títulos, foram excluídos 3.161 artigos, restando 60 estudos; com a realização da leitura dos resumos, foram excluídos 44 artigos, restando 16 e, depois de sua leitura na íntegra, 7 foram excluídos, restando 9 artigos, os quais apresentavam todos os critérios de elegibilidade, compondo a amostra final deste estudo (Figura 1).

As Tabelas 1 e 2 apresentam características metodológicas gerais dos 9 artigos selecionados para a revisão e dos processos de validação de instrumentos de autoavaliação em voz. Nenhum estudo eliminou ou acrescentou itens, quando comparados com os originais, validados internacionalmente.

Participaram das pesquisas de validação e adaptação cultural 1390 indivíduos, com média de 154,44 ( $\pm 82,40)$ por artigo, sendo que $7(77,8 \%)$ estudos contaram com um grupo de pacientes com queixas vocais e outro grupo sem queixa (controle) (Tabela 1).

Observou-se que a maioria dos artigos $(\mathrm{n}=6 ; 66,7 \%)$ englobou a adaptação cultural e linguística dos instrumentos para adequação ao português brasileiro e todos mencionaram se tratar de uma fase do processo de validação, realizada de acordo com as normas propostas pelo Scientific Advisory Commitee (SAC), do Medical Outcomes Trust (Comitê do Conselho Científico da Associação de Resultados Médicos) ${ }^{(23)}$. Qualidade de vida em voz e desvantagem vocal foram os construtos mais abordados nos protocolos validados (Tabela 1).

Em relação à população-alvo, apenas o Questionário Qualidade de Vida em Voz Pediátrico (QVV-P) é destinado a crianças e adolescentes. Os demais instrumentos foram estruturados para abrangerem, principalmente, adultos e idosos. Todos se destinaram a indivíduos de ambos os sexos e sensíveis a avaliar pessoas com disfonia ou sintomas vocais. Percebeu-se, ainda, que grande parte dos instrumentos $(\mathrm{n}=6 ; 66,7 \%)$ foi adaptada e validada na Região Sudeste do Brasil, principalmente no Estado de São Paulo (Tabela 1).

Os instrumentos foram compostos por um número entre 10 e 32 itens, todos com opções de resposta de acordo com a escala Likert, ou tipo Likert, com 5 ou mais possibilidades de resposta. Além disso, a maioria $(\mathrm{n}=7 ; 77,8 \%)$ possuía domínios relacionados ao construto (Tabela 2).

Em relação à obtenção dos escores, a fim de quantificar o traço latente avaliado, a forma de calcular foi a partir do somatório simples das respostas dos participantes para cada item, com exceção da escala adaptada para o português brasileiro URICA-V (University of Rhode Island Change Assessment) e do questionário Qualidade de Vida em Voz (QVV), cujos cálculos são diferenciados, que contam com fórmulas matemáticas e consideram os itens e seus domínios (Tabela 2). O QVV usa a fórmula clássica dos protocolos de avaliação de qualidade de vida. Apenas o Questionário Performance Vocal (QPV), a Escala de Sintomas Vocais (ESV) e a URICA-V apresentaram pontos de corte em seus estudos de validação, sendo que o deste último utilizou os valores referentes ao protocolo original (Tabela 2). Os pontos de corte dos outros instrumentos foram definidos em estudo posterior ${ }^{(17,28)}$, mas não na primeira publicação da escala em português, que foram os selecionados neste estudo.

Os domínios dos instrumentos, bem como os itens que os compõem, foram definidos de acordo com os protocolos na língua original, ou seja, não foi realizada análise fatorial adicional, em português brasileiro, para se confirmar a existência das mesmas subescalas ou domínios dos questionários. Apenas o Protocolo 
Tabela 1. Características gerais dos estudos selecionados para revisão sistemática e do processo de validação de instrumentos de autoavaliação em voz

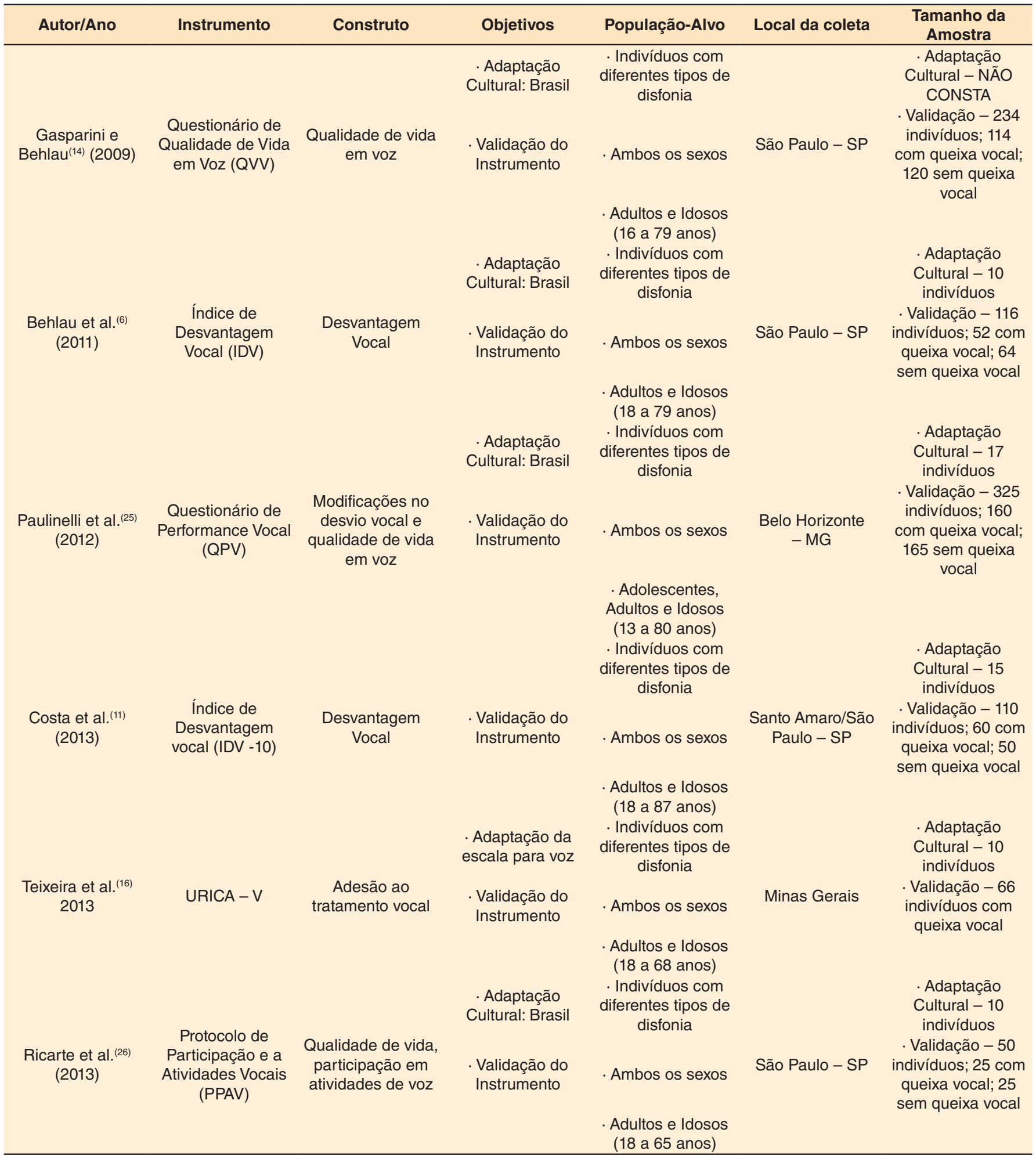

Legenda: URICA-V =adaptação brasileira da escala University of Rhode Island Change Assessment 
Tabela 1. Continuação...

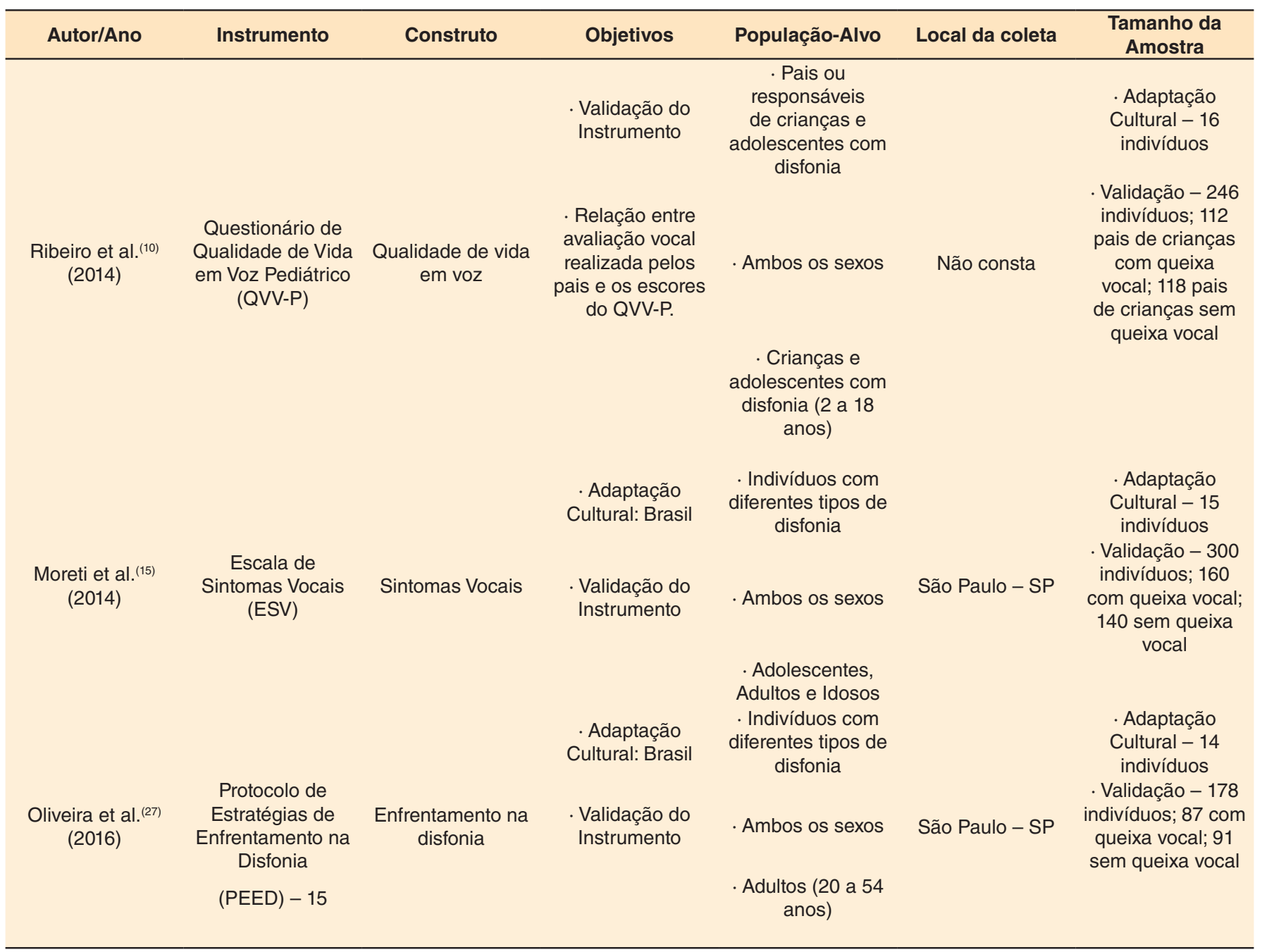

Legenda: URICA-V =adaptação brasileira da escala University of Rhode Island Change Assessment

de Estratégias de Enfrentamento na Disfonia (PEED-15) realizou análise fatorial confirmatória para verificar se houve mudança em relação aos itens e aos domínios, após adaptação cultural para o português brasileiro.

A estatística mais comum para análise dos instrumentos foi o coeficiente Alfa de Cronbach, que tem como finalidade avaliar sua confiabilidade. Apenas o artigo da escala URICA-V não utilizou esse teste (Tabela 3 ).

Utilizaram-se, principalmente, os testes de Wilcoxon e t-Student para monitoramento da sensibilidade dos questionários (escores) entre momentos pré e pós-terapia, também empregados na comparação intergrupos, caso e controle. Os testes ANOVA e Kruskal Wallis foram utilizados quando se compararam três ou mais grupos da população. Já para testar a sensibilidade dos itens, individualmente, um dos testes utilizados foi o dos Postos Sinalizados de Wilcoxon (Tabela 3).

Os testes de correlação de Pearson ou de Spearman foram citados (Tabela 3) como comprobatórios da validade dos dados, pois os escores extraídos dos instrumentos foram correlacionados com a autoavaliação vocal, bem como com a análise perceptivoauditiva da voz dos participantes e, à medida que essa correlação era positiva, consideravam-se válidas as informações dos instrumentos.
Constatou-se que os estudos de validação dos protocolos QVV-P e a ESV utilizaram análise perceptivoauditiva da voz para fazer correlação com seus escores. O QVV, QPV, IDV-10, IDV-30 e PPAV consideraram a autoavaliação vocal e o PEED-15 utilizou tanto a análise perceptivoauditiva, quanto a autoavaliação da qualidade da voz (entre excelente, muito boa, boa, razoável ou ruim) para correlacionar com os escores do protocolo e verificar sua validade.

Os instrumentos QVV, URICA-V e PEED-15 não apresentaram testes de reaplicação após o tratamento e foram indicados apenas para avaliação. Os demais foram testados e classificados como sensíveis à aplicação nos momentos de avaliação, monitoramento e reavaliação após a reabilitação vocal.

Os autores dos estudos selecionados concluíram que os protocolos propostos são válidos e sensíveis, de modo que fornecem dados de autoavaliação confiáveis (Tabela 3). Observou-se que a metade dos instrumentos de autoavaliação vocal $(n=5,55,6 \%)$ seguiu todas as etapas propostas para validação, de acordo com as normas propostas pelo Comitê do Conselho Científico da Associação de Resultados Médicos (Scientific Advisory Commitee of the Medical Outcomes Trust $)^{(23)}$ para o processo de validação de instrumentos. De forma geral, a etapa de validação menos abordada nos estudos foi a de sensibilidade (Tabela 4). 
Tabela 2. Características de obtenção dos escores e estrutura de instrumentos de autoavaliação vocal validados para o português brasileiro

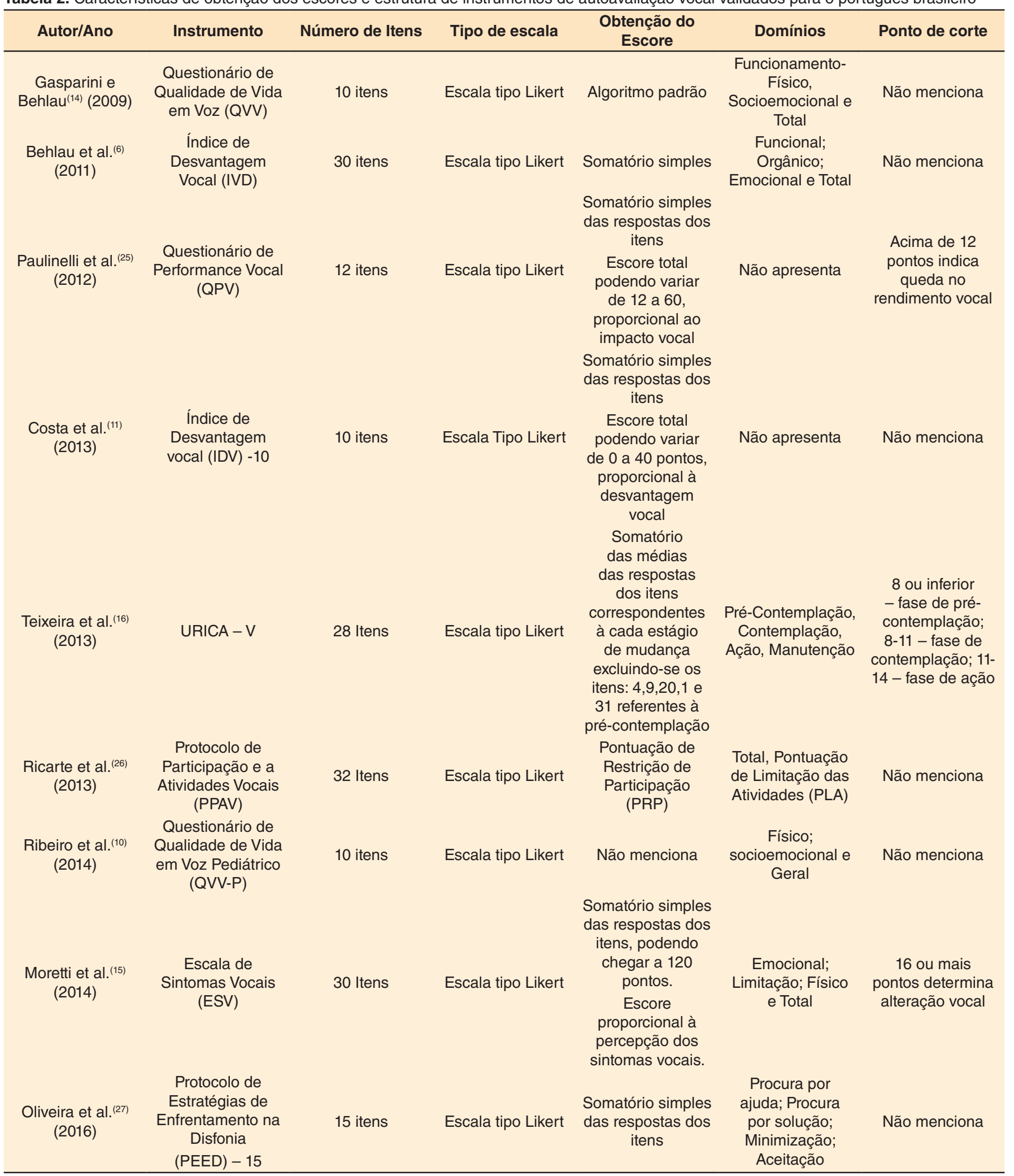

Legenda: URICA-V =adaptação brasileira da escala University of Rhode Island Change Assessment

\section{DISCUSSÃO}

A presente revisão reuniu estudos que objetivaram adaptar culturalmente e validar os protocolos de autoavaliação no Brasil até o momento, e que são utilizados na prática clínica e em pesquisas, tais como: Questionário de Qualidade de Vida em Voz - QVV (14), para mensurar impacto negativo na qualidade de vida, em virtude da disfonia; Índice de Desvantagem 
Tabela 3. Características do processo de validação dos instrumentos de autoavaliação em voz

\begin{tabular}{ccc}
\hline Autor/Ano & Instrumento & Análise Estatística \\
\hline & & \\
Gasparini e Behlau(14) & $\begin{array}{c}\text { Questionário de Qualidade } \\
\text { de Vida em Voz (QVV) }\end{array}$ & $\begin{array}{c}\text { Teste Kruskal-Wallis; } \\
\text { Correlação Alfa de } \\
\text { Cronbach; Teste de } \\
\text { Wilcoxon }\end{array}$
\end{tabular}

Behlau et al. ${ }^{(6)}(2011)$

Paulinelli et al. ${ }^{(25)}$ (2012)

Costa et al. ${ }^{(11)}(2013)$

Teixeira et al. ${ }^{(16)}$ (2013)

Ricarte et al. ${ }^{(26)}$ (2013)

Ribeiro et al. ${ }^{(10)}(2014)$
Índice de Desvantagem Vocal (IDV)

Questionário de Performance Vocal (QPV)

Indice de Desvantagem vocal (IDV) -10

$$
\text { URICA - V }
$$

Protocolo de Participação e a Atividades Vocais (PPAV)

Questionário de Qualidade de Vida em Voz Pediátrico (QVV-P)
Teste Kruskal Wallis; Teste Wilcoxon; Correlação Alfa de Cronbach

Teste de Mann-Whitney; Correlação de Spearman; Teste de Wilcoxon; Alfa de Cronbach

Correlação de Spearman; Teste dos Postos sinalizados de Wilcoxon; Correlação Alfa de Cronbach

Correlação de Spearman; Teste dos Postos Sinalizados de Wilcoxon; Alfa de Cronbach

Qui-Quadrado; Análise de Variância - ANOVA

Teste Qui-Quadrado; Teste de Mann-Whitney; Teste de McNemar ; Teste dos Postos Sinalizados de Wilcoxon; Correlação de Spearman; Razão de Verossimilhança; Alfa de Cronbach
Avaliação e monitoramento do tratamento (16 participantes)

Avaliação e monitoramento do tratamento (10 participantes)

Avaliação e monitoramento do tratamento (39 participantes)

Avaliação e monitoramento do tratamento (21 participantes)

Avaliação e monitoramento do tratamento (50 participantes)

Avaliação e monitoramento do tratamento (16 participantes)
Conclusão

O QVV avalia

especificamente pacientes com problemas de voz.

É válido, confiável e responsivo à mudança.

A versão brasileira pode ser proposta como avaliação da qualidade de vida de pacientes disfônicos e tratamento.

O IDV é válido, confiável e responsivo às medidas de avaliação da mudança relacionadas à autopercepção da desvantagem vocal. Pode ser utilizado para avaliação de pacientes disfônicos e para os resultados do tratamento.

O QPV mostrou-se válido, confiável, reprodutivo e sensível ao tratamento, podendo ser considerado como mais uma opção para relacionar qualidade de vida com problema de voz.

O IDV-10 é validado para o português brasileiro, com propriedades psicométricas de validade, confiabilidade e sensibilidade comprovadas para o emprego em indivíduos com problemas de voz.

O uso da escala URICA-V revelou que a maioria dos pacientes com disfonia em tratamento se encontra no estágio de contemplação, o que pode restringir os resultados da terapia. Não se constatou relação entre variáveis demográficas e estágios de adesão do instrumento

A versão brasileira do protocolo (PPAV) é válida, confiável e sensível especificamente para avaliação da qualidade de vida de indivíduos com alterações vocais e os resultados dos tratamentos.

O QVV-P está validado para o português

brasileiro, apresentando equivalência cultural e medidas psicométricas de validade, confiabilidade e sensibilidade, testadas de forma satisfatória. 
Tabela 3. Continuação...

\begin{tabular}{|c|c|c|c|c|}
\hline Autor/Ano & Instrumento & Análise Estatística & Aplicação Testada & Conclusão \\
\hline Moretti et al. ${ }^{(15)}$ (2014) & $\begin{array}{l}\text { Escala de Sintomas Vocais } \\
\text { (ESV) }\end{array}$ & $\begin{array}{l}\text { Teste t-Student; Correlação } \\
\text { Alfa de Crombach }\end{array}$ & $\begin{array}{l}\text { Triagem, avaliação } \\
\text { e monitoramento } \\
\text { do tratamento ( } 86 \\
\text { participantes) }\end{array}$ & $\begin{array}{l}\text { A ESV é um instrumento } \\
\text { válido, confiável para } \\
\text { avaliação da voz e dos } \\
\text { sintomas vocais. } \\
\text { Discrimina indivíduos com } \\
\text { voz saudável e disfônicos, } \\
\text { confirmando utilização } \\
\text { desse instrumento na } \\
\text { triagem em populações de } \\
\text { alto risco. }\end{array}$ \\
\hline Oliveira et al. ${ }^{(27)}(2016)$ & $\begin{array}{l}\text { Protocolo de Estratégias } \\
\text { de Enfrentamento na } \\
\text { Disfonia (PEED) - 15 / } \\
\text { Enfrentamento na disfonia }\end{array}$ & $\begin{array}{c}\text { Análise Fatorial } \\
\text { confirmatória; Análise } \\
\text { de Variância - ANOVA; } \\
\text { Correlação de Pearson; } \\
\text { Teste de esfericidade de } \\
\text { Bartlett; Índice de Kaiser- } \\
\text { Meyer-Olkin; Correlação } \\
\text { Alfa de Cronbach }\end{array}$ & Avaliação & $\begin{array}{l}\text { O PEED-15 passou } \\
\text { por adaptação cultural } \\
\text { e validação de forma } \\
\text { efetiva e é um instrumento } \\
\text { específico para avaliação } \\
\text { de pacientes com } \\
\text { problemas de voz. }\end{array}$ \\
\hline
\end{tabular}

Legenda: URICA-V =adaptação brasileira da escala University of Rhode Island Change Assessment

Vocal - IDV ${ }^{(6)}$, para mensurar a percepção de desvantagem decorrente da manifestação da disfonia; Questionário de Performance Vocal - QPV ${ }^{(25)}$ e Protocolo de Participação e a Atividades Vocais - $\operatorname{PPAV}^{(26)}$, a fim de observar a comunicação do indivíduo; URICA - $\mathrm{V}^{(16)}$, adaptada para avaliar o estágio de adesão do paciente com disfonia ao tratamento; Questionário de Qualidade de Vida em Voz Pediátrico - QVV-P(10), Escala de Sintomas Vocais - ESV ${ }^{(15)}$, para investigar a ocorrência de sintomas laríngeos, vocais e ainda percepção de limitações devido à disfonia; Protocolo de Estratégias de Enfrentamento na Disfonia - PEED-15(27), com o objetivo de avaliar de que forma o indivíduo está lidando com a disfonia.

Foi analisado cada processo de validação, visto que a garantia da confiabilidade de um instrumento deve ser embasada por meio do planejamento e observância de todos os procedimentos ${ }^{(29)}$ e que muitos protocolos relacionados à autoavaliação vocal consideraram este planejamento em sua validação.

Os artigos de validação de instrumentos em saúde tendem a se planejar de acordo com as principais etapas de validação do Scientific Advisory Committee (SAC), do Medical Outcomes Trust $^{(23)}$, as mais utilizadas no Brasil atualmente, embora não seja a única recomendação. São previstas oito etapas: (1) modelo conceitual e de medida, (2) confiabilidade, (3) validade, (4) sensibilidade, (5) interpretabilidade, (6) demanda de administração e resposta, (7) modos alternativos de aplicação e (8) adaptação linguística e cultural.

Assim, a primeira (1) etapa está relacionada ao modelo conceitual e de medida, que torna o instrumento mais racional, incluindo dados da população-alvo e informação de especialistas, caracterizando o desenvolvimento do instrumento, permitindo sua adequação para outra língua e cultura. A segunda (2) e a terceira (3) etapas atêm-se à confiabilidade, que mede a obtenção de dados fidedignos, por meio da análise da consistência interna, com uso do Alfa de Cronbach, e reprodutibilidade, aplicação do teste-reteste, além da busca de evidências de validade, que certificam se o instrumento mede o que se propõe a medir, respectivamente ${ }^{(23)}$.

A quarta (4) etapa mede a sensibilidade do teste em detectar mudanças longitudinais, comparando grupos em momentos pré e pós-terapia, por exemplo. Essa etapa é seguida pela interpretabilidade (5), medida pela qual pode se oferecer um significado qualitativo para a pontuação obtida nos escores, que é complementar à sexta (6) regra relacionada às formas alternativas de resposta e administração do questionário, como tempo de aplicação, nível de leitura, compreensão, energia e outros requisitos relacionados à população, quanto ao instrumento ${ }^{(23)}$.

A sétima (7) etapa corresponde aos modos alternativos de aplicação, incluindo instrumentos aplicáveis pelo avaliador, autoaplicáveis, ou aplicados por meio do computador, por exemplo. A última etapa (8) corresponde à adaptação linguística e cultural, a mais realizada nos artigos revisados no presente estudo, em que é feita a tradução e adaptação dos instrumentos de autoavaliação vocal para o português brasileiro.

Geralmente, na descrição da oitava etapa, os estudos relataram que foi realizada por fonoaudiólogos bilíngues e um tradutor profissional, não envolvidos nas etapas anteriores ${ }^{(23)}$. Posteriormente, as versões traduzidas foram analisadas e modificadas por consenso, por outros fonoaudiólogos especialistas em voz e fluentes no inglês. $O$ instrumento traduzido ainda foi aplicado em um grupo de indivíduos pertencentes à populaçãoalvo, a fim de identificar o nível de clareza e compreensão de cada item. Caso necessário, foram realizadas alterações até chegar ao instrumento final ${ }^{(6,10,14,16,25-27,30)}$.

Para a elaboração de um instrumento de medidas, faz-se necessário definir o que deve ser medido e como deve ser realizada essa mensuração, a partir da determinação da sua validade. A validade refere-se à capacidade que os métodos utilizados numa pesquisa proporcionam à consecução fidedigna de seus objetivos e, consequentemente, à característica se de fato um teste está mensurando alguns atributos reais que, supostamente, está mensurando. A validade é a medida verdadeira que consiste no quão útil é o teste ${ }^{(31,32)}$.

Validade, portanto, não é um conceito unitário e existem várias formas para avaliá-la, cada uma com maior pertinência, em razão do objetivo e contextos em que se pretende utilizar o instrumento de avaliação. Pode ser estimada por meio dos seguintes métodos: validade de conteúdo, validade relacionada a um critério e validade do construto ${ }^{(31)}$.

Os protocolos da área de voz foram validados para o português brasileiro, publicados nos periódicos e, posteriormente 
Tabela 4. Instrumentos validados de acordo com os requisitos do Scientific Advisory Committee, do Medical Outcomes Trust

\begin{tabular}{|c|c|c|c|c|c|c|c|c|c|}
\hline \multirow[b]{2}{*}{ Autor/Ano } & \multirow[b]{2}{*}{$\begin{array}{l}\text { Instrumento } \\
\text { / Construto }\end{array}$} & \multicolumn{8}{|c|}{ Etapas do Comitê } \\
\hline & & $\begin{array}{c}\text { Etapa } 1 \\
\text { (Modelo } \\
\text { conceitual } \\
\text { e de } \\
\text { medida) } \\
\end{array}$ & $\begin{array}{l}\text { Etapa } 2 \\
\text { (Confiabi- } \\
\text { lidade) }\end{array}$ & $\begin{array}{c}\text { Etapa } 3 \\
\text { (Validade) }\end{array}$ & $\begin{array}{c}\text { Etapa } 4 \\
\text { (Sensibi- } \\
\text { lidade) }\end{array}$ & $\begin{array}{c}\text { Etapa } 5 \\
\text { (Interpre- } \\
\text { tabilidade) }\end{array}$ & $\begin{array}{c}\text { Etapa } 6 \\
\text { (Forma de } \\
\text { adminis- } \\
\text { tração e } \\
\text { resposta) }\end{array}$ & $\begin{array}{c}\text { Etapa } 7 \\
\text { (Modo de } \\
\text { aplicação) }\end{array}$ & $\begin{array}{c}\text { Etapa } 8 \\
\text { (Adaptação } \\
\text { cultural e } \\
\text { linguística) }\end{array}$ \\
\hline $\begin{array}{c}\text { Gasparini } \\
\text { e Behlau } \\
(2009)\end{array}$ & $\begin{array}{c}\text { Questionário } \\
\text { de } \\
\text { Qualidade } \\
\text { de Vida em } \\
\text { Voz - QVV/ } \\
\text { Qualidade } \\
\text { de vida em } \\
\text { voz }\end{array}$ & $x$ & $x$ & $x$ & -- & $x$ & $x$ & $\mathrm{x}$ & -- \\
\hline $\begin{array}{l}\text { Behlau et al. } \\
\text { (6) (2011) }\end{array}$ & $\begin{array}{c}\text { Índice de } \\
\text { Desvantagem } \\
\text { Vocal/ IDV - } \\
\text { Desvantagem } \\
\text { Vocal }\end{array}$ & $x$ & $\mathrm{X}$ & $\mathrm{X}$ & $\mathrm{x}$ & $x$ & -- & $\mathrm{x}$ & $x$ \\
\hline $\begin{array}{l}\text { Paulinelli et al. } \\
\text { (25) }(2012)\end{array}$ & $\begin{array}{c}\text { Questionário } \\
\text { de } \\
\text { Performance } \\
\text { Vocal - } \\
\text { QPV / } \\
\text { Modificações } \\
\text { no Desvio } \\
\text { Vocal e } \\
\text { qualidade } \\
\text { de vida em } \\
\text { voz }\end{array}$ & $\mathrm{X}$ & $\mathrm{X}$ & $\mathrm{X}$ & $x$ & $\mathrm{X}$ & $x$ & $\mathrm{x}$ & $x$ \\
\hline $\begin{array}{l}\text { Costa et al. } \\
\text { (11) }(2013)\end{array}$ & $\begin{array}{c}\text { Indice de } \\
\text { Desvantagem } \\
\text { vocal - } \\
\text { IDV-10 / } \\
\text { Desvantagem } \\
\text { Vocal }\end{array}$ & $x$ & $\mathrm{x}$ & $x$ & $x$ & $x$ & $x$ & $x$ & $x$ \\
\hline $\begin{array}{l}\text { Texeira et al. } \\
\text { (16) }(2013)\end{array}$ & $\begin{array}{c}\text { URICA - V/ } \\
\text { Adesão ao } \\
\text { tratamento } \\
\text { vocal }\end{array}$ & $\mathrm{x}$ & - & $x$ & -- & $x$ & $x$ & $\mathrm{x}$ & -- \\
\hline $\begin{array}{l}\text { Ricarte et al. } \\
\text { (26) }(2013)\end{array}$ & $\begin{array}{c}\text { Protocolo de } \\
\text { Participação } \\
\text { e a } \\
\text { Atividades } \\
\text { Vocais - } \\
\text { PPAV / } \\
\text { Qualidade } \\
\text { de vida, } \\
\text { participação } \\
\text { em } \\
\text { atividades } \\
\text { de voz }\end{array}$ & $x$ & $x$ & $\mathrm{X}$ & $\mathrm{x}$ & $x$ & $x$ & $x$ & $x$ \\
\hline $\begin{array}{l}\text { Ribeiro et al. } \\
\text { (10) }(2014)\end{array}$ & $\begin{array}{c}\text { Questionário } \\
\text { de } \\
\text { Qualidade } \\
\text { de Vida } \\
\text { em Voz } \\
\text { Pediátrico } \\
\text { - QVV-P/ } \\
\text { Qualidade } \\
\text { de vida em } \\
\text { voz }\end{array}$ & $x$ & $x$ & $x$ & $x$ & $x$ & $x$ & $x$ & $x$ \\
\hline
\end{tabular}

Legenda: URICA-V =adaptação brasileira da escala University of Rhode Island Change Assessment 
Tabela 4. Continuação...

\begin{tabular}{|c|c|c|c|c|c|c|c|c|c|}
\hline \multirow[b]{2}{*}{ Autor/Ano } & \multirow[b]{2}{*}{$\begin{array}{l}\text { Instrumento } \\
\text { / Construto }\end{array}$} & \multicolumn{8}{|c|}{ Etapas do Comitê } \\
\hline & & $\begin{array}{c}\text { Etapa } 1 \\
\text { (Modelo } \\
\text { conceitual } \\
\text { e de } \\
\text { medida) } \\
\end{array}$ & $\begin{array}{l}\text { Etapa } 2 \\
\text { (Confiabi- } \\
\text { lidade) }\end{array}$ & $\begin{array}{c}\text { Etapa } 3 \\
\text { (Validade) }\end{array}$ & $\begin{array}{l}\text { Etapa } 4 \\
\text { (Sensibi- } \\
\text { lidade) }\end{array}$ & $\begin{array}{c}\text { Etapa } 5 \\
\text { (Interpre- } \\
\text { tabilidade) }\end{array}$ & $\begin{array}{c}\text { Etapa } 6 \\
\text { (Forma de } \\
\text { adminis- } \\
\text { tração e } \\
\text { resposta) }\end{array}$ & $\begin{array}{c}\text { Etapa } 7 \\
\text { (Modo de } \\
\text { aplicação) }\end{array}$ & $\begin{array}{c}\text { Etapa } 8 \\
\text { (Adaptação } \\
\text { cultural e } \\
\text { linguística) }\end{array}$ \\
\hline $\begin{array}{l}\text { Moreti et al. } \\
\text { (15) (2014) }\end{array}$ & $\begin{array}{c}\text { Escala de } \\
\text { Sintomas } \\
\text { Vocais } \\
\text { - ESV/ } \\
\text { Sintomas } \\
\text { Vocais }\end{array}$ & $\mathrm{x}$ & $\mathrm{X}$ & $\mathrm{x}$ & $\mathrm{x}$ & $\mathrm{x}$ & $\mathrm{x}$ & $x$ & $X$ \\
\hline $\begin{array}{l}\text { Oliveira et al. } \\
\text { (27) (2016) }\end{array}$ & $\begin{array}{l}\text { Protocolo de } \\
\text { Estratégias } \\
\text { de } \\
\text { Enfrentamento } \\
\text { na Disfonia - } \\
\text { PEED - 15/ } \\
\text { Enfrentamento } \\
\text { na disfonia }\end{array}$ & $\mathrm{x}$ & $\mathrm{x}$ & $\mathrm{X}$ & -- & $\mathrm{X}$ & $\mathrm{X}$ & $\mathrm{x}$ & $\mathrm{X}$ \\
\hline
\end{tabular}

Legenda: URICA-V =adaptação brasileira da escala University of Rhode Island Change Assessment

utilizados em pesquisas observacionais e de intervenção, que têm a finalidade de produzir evidências científicas sobre o aspecto vocal estudado (construto), nas mais diversas populações e regiões do país. Esse fato deve ser considerado no momento da adaptação cultural, tendo em vista que o Brasil é um país com vasto território e tem uma população diversificada, com características culturais e sociodemográficas diferenciadas. Ressalta-se, então, a importância de que a adaptação de um instrumento de autoavaliação envolva indivíduos de diversas regiões do país, a fim de maior abrangência cultural e consequente melhor aplicação e aceitação entre profissionais e pessoas com problemas vocais.

Além disso, sabe-se que protocolos desenvolvidos em determinada cultura valem especificamente para aquela realidade e, caso precisem ser utilizados em situações diferentes, é sugerido que se realize outro processo de validação ${ }^{(25-27,29)}$, considerando não apenas a língua, mas as características populacionais e culturais do país. Nesse processo, o instrumento deve ser aplicado com a população-alvo para posterior análise das respostas e obtenção dos cálculos dos escores, de modo que se possam inferir as medidas psicométricas para outras amostras com caracterização semelhante.

O tipo de modelagem estatística utilizado na análise das medidas psicométricas dos instrumentos de autoavaliação vocal selecionados foi a Teoria Clássica de Teste (TCT), que é mais tradicional e comumente utilizada em estudos de validação. Nesse modelo, o nível do atributo/escore é dado com o somatório das respostas em cada um dos itens, como observado nos cálculos sugeridos nos estudos que validaram questionários na área de voz.

Esse método, porém, padece de limitações, pois seus parâmetros são generalistas, considerando valores padrão da escala de resposta dos itens, além de depender diretamente da amostra de indivíduos estudada, de tal modo que os resultados para um mesmo teste podem variar de acordo com a amostra ${ }^{(33,34)}$, o que reforça a ideia de que não é recomendado realizar inferências a partir de escores de testes realizados em populações diferentes.

Apesar da TCT ter grande importância e utilidade, alguns autores têm proposto a aplicação de teorias mais modernas, devido as suas limitações, como a Teoria de Resposta ao Item (TRI), que se sobressai em relação à $\mathrm{TCT}^{(35,36)}$. A TRI possibilita trabalhar com a classificação do indivíduo em relação ao traço latente e analisa item a item do instrumento, o que proporciona maior aproveitamento da informação contida em cada um, ao lhes dar diferentes pesos, de acordo com sua importância, de tal forma que melhora a sensibilidade na medida do traço latente ${ }^{(22,34,37)}$.

O questionário da área de voz mais validado e adaptado para diversas línguas/culturas é o Índice de Desvantagem Vocal (IDV). Revisão sistemática realizada com o objetivo de investigar a validação de diferentes versões do IDV-30, em relação a sua validade, confiabilidade e processo de tradução encontrou esse protocolo traduzido em 11 línguas. Foi considerado confiável, com boa consistência interna, mas com validade de construção moderada no que diz respeito aos seus domínios ${ }^{(38)}$.

O artigo publicado em $2007^{(33)}$ já pontuava as limitações referentes à modelagem clássica de cálculos (TCT), como o fato de a validade e confiabilidade serem dependentes da amostra, além da questão da determinação dos fatores do IDV, que apresentou três domínios na versão original em inglês americano. Porém, outros estudos encontraram dois ${ }^{(39)} \mathrm{e}$ até quatro domínios, quando realizada a análise fatorial confirmatória ${ }^{(40)}$ em populações diferentes. O estudo citado aplicou a TRI a partir do Modelo de Rasch, no IDV-30, e concluiu que a análise item a item tornou o protocolo mais adequado, não apenas na avaliação inicial do paciente, mas também para avaliar a eficácia da terapia.

Além desse artigo, outros estudos destacaram as vantagens da TRI e sugeriram que a nova proposta fosse aplicada nas validações dos questionários de autoavaliação vocal ${ }^{(30,36,39)}$. Sugere-se, então, que essa nova proposta de cálculos seja considerada não apenas nas novas validações, mas também aplicada nos protocolos preexistente, como os descritos nesta revisão $0^{(33)}$.

Observou-se que, apenas na validação do $\operatorname{PEED}^{(27)}$, foi realizada análise fatorial confirmatória, em que os itens corresponderam a quatro fatores/domínios: procura por ajuda, procura por solução, minimização e aceitação, diferentemente dos domínios apontados no estudo original, cujo instrumento 
considera apenas dois domínios: foco no problema e foco na emoção ${ }^{(27)}$. Apesar disso, na adaptação do PEED para o português brasileiro, foram considerados os dois domínios herdados da versão na língua inglesa. Os demais estudos utilizaram os fatores dos protocolos originais. A escala URICA-V ${ }^{(16)}$ utilizou, ainda, os pontos de corte da escala original, ou seja, inespecíficos para a área de voz e para a população brasileira.

É interessante reforçar a importância da realização da Análise Fatorial Exploratória (AFE) e da Análise Fatorial Confirmatória (AFC), a fim de verificar se, na população brasileira, ou em uma população específica, o número de fatores é semelhante ao do instrumento validado em outro contexto, ou se os itens possuem a mesma relação com os domínios predeterminados ${ }^{(41)}$.

Assim, os instrumentos inicialmente direcionados a indivíduos com faixas etárias específicas e que avaliam momentos pontuais do tratamento podem se tornar mais abrangentes a partir da análise fatorial e aplicação da TRI, tendo em vista que será considerada a aptidão do indivíduo em relação ao aspecto vocal investigado.

Outra etapa normatizada é a de validação propriamente, cujo objetivo é que o instrumento traduzido seja compatível ao original, em relação à equivalência linguística, conceitual e psicométrica e às medidas de confiabilidade e sensibilidade, por exemplo, bem como que ele meça o que se propõe a medir ${ }^{(29)}$.

Como medidas de validação, foi comum os estudos apresentarem correlações (Pearson e Spearman) entre os escores dos questionários e a autopercepção da $\mathrm{Voz}^{(10,15,25-27)}$, que tem sido mais valorizada, e/ou avaliação clínica perceptivoauditiva ${ }^{(42)}$, que é o padrão de referência mais utilizado na área de Fonoaudiologia, para comprovar sua sensibilidade. Essas medidas possibilitam, também, avaliar a sensibilidade e especificidade dos questionários. A medida da confiabilidade/fidedignidade também é uma etapa do processo de validação e é a capacidade que o teste/item tem de reproduzir um resultado consistente, mesmo sendo analisado por diferentes avaliadores ou momentos - medida por meio do coeficiente Alfa de Cronbach ${ }^{(29)}$.

A sensibilidade é a capacidade de o teste identificar diferença nos escores entre grupos de pacientes com diferentes tipos de disfonia, que foi medida pelos testes t-Student e Wilcoxon para duas amostras independentes e Kruskal Wallis e ANOVA para mais de duas amostras independentes, nos casos descritos neste estudo. Outra medida importante no processo de validação é a responsividade, que verifica se o instrumento detecta mudanças nas condições dos pacientes, em relação ao traço latente, como ocorre na aplicação pré e pós-terapia, realizada por meio de testes pareados em alguns dos estudos selecionados ${ }^{(29,43)}$. Sugere-se que sejam feitas essas mensurações, importantes no processo de validação, em estudos futuros, utilizando esses protocolos.

A URICA-V e o PEED-15 não apresentaram dados de responsividade e, por isso, são indicados apenas na avaliação inicial. Seria interessante verificar a sensibilidade desses dois instrumentos ao processo de reabilitação, principalmente para observar se houve deslocamento do estágio do ciclo de mudança para, pelo menos, o de ação, ou, preferencialmente, o de manutenção na escala URICA-V ${ }^{(44)}$ e se houve o desenvolvimento de estratégias de enfrentamento mais funcionais para lidar com uma possível recorrência do problema no PEED-15.

Apesar de a escala URICA-V não ser indicada para o monitoramento no artigo de validação ${ }^{(16)}$, estudos ${ }^{(45,46)}$ utilizaramna para observar modificação do estado de prontidão pré e pósterapia de grupo e individual. Não foram encontrados resultados significativos em relação à sensibilidade e responsividade do protocolo, visto que não se observaram diferenças na prontidão entre os momentos de terapia, em nenhuma das modalidades terapêuticas (terapia individual e de grupo), mesmo com a melhora dos indivíduos melhorado em outros aspectos ${ }^{(45,46)}$.

Além disso, um estudo ${ }^{(47)}$ investigou a precisão dos domínios existentes na escala URICA-V por meio de análise fatorial confirmatória. Verificou-se que alguns itens do questionário não apresentaram correlação significativa referente ao próprio domínio de origem, resultados que permitiram propor um ajuste na escala URICA-V para refinar a habilidade do instrumento no momento da avaliação.

Tendo em vista o exposto, as pesquisas selecionadas buscaram seguir as normas de validação, mas, apesar disso, a maioria dos processos de validação tem alguma lacuna a ser mais bem observada e solucionada, sobretudo em relação às medidas psicométricas. Essa observação reafirma os achados de uma revisão sistemática realizada em $2010^{(36)}$, que analisou os protocolos de autoavaliação relacionados à disfonia, a partir das normas do Scientific Advisory Committee (SAC), do Medical Outcome Trust ${ }^{(23)}$, e pôde concluir que houve problemas relacionados ao desenvolvimento e deficit nas propriedades psicométricas dos instrumentos originais.

De modo geral, os questionários validados nos artigos selecionados para a presente revisão obtiveram bons resultados nos testes estatísticos propostos para cada etapa, principalmente aqueles que seguiram, efetivamente, a maioria das etapas, tanto que a maior parte concluiu que os protocolos propostos são válidos, sensíveis e fornecem dados de autoavaliação confiáveis. Apesar disso, observou-se que não houve evolução em relação à análise dos instrumentos, mas uma padronização dos métodos, sendo, geralmente, utilizados os mesmos modelos estatísticos. Sugere-se, então, que estudos sejam realizados, com o objetivo de aplicar métodos contemporâneos, como a TRI, nesses instrumentos ${ }^{(36,47)}$, com a finalidade de melhorar suas medidas psicométricas de discriminação, sensibilidade, confiabilidade e responsividade, além de se obter parâmetros referentes aos itens e a sua real contribuição para entendimento da instalação, manutenção e reabilitação da disfonia.

Vale salientar que existem outros instrumentos de autoavaliação vocal, geralmente elaborados em inglês e traduzidos/validados para o português brasileiro, além dos discutidos no presente artigo. Eles não foram contemplados nesta revisão por não terem sido localizados na pesquisa, a partir da estratégia de busca, sobretudo da combinação dos descritores utilizados, ou por não satisfazerem aos critérios de elegibilidade, mas não são menos importantes por isso. Alguns exemplos são: Escala de Desconforto do Trato Vocal (EDTV) ${ }^{(48)}$, Protocolo de Estratégias de Enfrentamento na Disfonia (PEED-27) ${ }^{(18)}$, Questionário de Autoavaliação Vocal para Transexuais de Homem para Mulher $(T V Q)^{(49)}$, entre outros. Esses instrumentos possuem características de validação semelhantes às dos protocolos descritos e, por isso, as sugestões discutidas no presente estudo podem se estender aos demais protocolos de autoavaliação vocal.

Dessa forma, o avanço das ciências computacional, estatística e psicométrica, bem como da área de voz, tem permitido o aprimoramento de métodos e os tornado naturalmente acessíveis, direcionando um novo olhar para os questionários de autoavaliação, desde sua elaboração até a revisão de suas medidas psicométricas ${ }^{(36,47)}$. O aprofundamento sobre as etapas a serem cumpridas e sobre os métodos contemporâneos de análise deve ser considerado desde o primeiro momento da elaboração, ou adaptação cultural de um instrumento de autoavaliação vocal, 
bem como na normatização de instrumentos preexistentes, como os apresentados no presente estudo. As reflexões aqui expostas podem auxiliar pesquisadores a nortear esses processos.

\section{CONCLUSÃO}

Foram encontrados nove artigos referentes a protocolos de autoavaliação de um problema de voz, validados para a população brasileira. A validação dos estudos buscou seguir as normas propostas internacionalmente. Os construtos mais abordados pelos instrumentos foram qualidade de vida em voz e índice de desvantagem vocal. Os protocolos abrangeram todas as faixas etárias do ciclo vital, principalmente a de adultos e idosos, ambos os sexos, e eram destinados a indivíduos com disfonia/queixas vocais.

\section{REFERÊNCIAS}

1. Parque K, Behlau M. Perda de voz em professores e não professores. Rev Soc Bras Fonoaudiol. 14(4):463-9.

2. Simberg S, Santtila P, Soveri A, Varjonen M, Sala E, Sandnabba NK. Exploring genetic and environmental effects in dysphonia: a twin study. J Speech Lang Hear Res. 2009;52(1):153-63. http://dx.doi. org/10.1044/1092-4388(2008/07-0095). PMid:18664695.

3. Solomon NP, Helou LB, Makashay MJ, Stojadinovic A. Aerodynamic evaluation of the postthyroidectomy voice. J Voice. 2012;26(4):45461. http://dx.doi.org/10.1016/j.jvoice.2011.03.010. PMid:21782386.

4. Ruotsalainen J, Sellman J, Lehto L, Verbeek J. Systematic review of the treatment of functional dysphonia and prevention of voice disorders. Otolaryngol Head Neck Surg. 2008;138(5):557-65. http:// dx.doi.org/10.1016/j.otohns.2008.01.014. PMid:18439458.

5. Dejonckere PH, Bradley P, Clemente P, Cornut G, Crevier-Buchman L, Friedrich $\mathrm{G}$, et al. A basic protocol for functional assessment of voice pathology, especially for investigating the efficacy of (phonosurgical) treatments and evaluating new assessment techniques. Guideline elaborated by the Committee on Phoniatrics of the European Laryngological Society (ELS). Eur Arch Otorhinolaryngol. 2001;258(2):77-82. http:// dx.doi.org/10.1007/s004050000299. PMid:11307610.

6. Behlau M, Alves Dos Santos Lde M, Oliveira G. Cross-cultural adaptation and validation of the voice handicap index into Brazilian Portuguese. J Voice. 2011;25(3):354-9. http://dx.doi.org/10.1016/j. jvoice.2009.09.007. PMid:20434874.

7. Freitas SAVSF, Ferreira AJS. Avaliação acústica e áudio perceptiva na caracterização da voz humana [tese]. Porto, Portugal: Universidade do Porto; 2012.

8. WHO: World Health Organization. Measuring quality of life. The world health organization quality of life instruments. Geneva: WHO/ MSA/MNH/PSF; 1997. p. 1-15.

9. Moreti F, Zambon F, Oliveira G, Behlau M. Cross-cultural adaptation of the Brazilian version of the Voice Symptom Scale: VoiSS. J Soc Bras Fonoaudiol. 2011 Dez;23(4):398-400. http://dx.doi.org/10.1590/ S2179-64912011000400018. PMid:22231064.

10. Ribeiro LL, Paula KM, Behlau M. Voice-related quality of life in the pediatric population: validation of the Brazilian version of the Pediatric Voice-Related Quality-of Life survey. Codas. 2014 Jan-Fev;26(1):87-95. http://dx.doi.org/10.1590/s2317-17822014000100013. PMid:24714864.
11. Costa T, Oliveira G, Behlau M. Validation of the Voice Handicap Index: 10 (VHI-10) to the Brazilian Portuguese. CoDAS. 2013 Out;25(5):482-5. http://dx.doi.org/10.1590/S2317-17822013000500013. PMid:24408554.

12. Ugulino AC, Oliveira G, Behlau M. Perceived dysphonia by the clinician's and patient's viewpoint. J Soc Bras Fonoaudiol. 2012;24(2):113-8. http://dx.doi.org/10.1590/S2179-64912012000200004. PMid:22832676.

13. Karnell MP, Melton SD, Childes JM, Coleman TC, Dailey SA, Hoffman HT. Reliability of clinician-based (GRBAS and CAPE-V) and patientbased (V-RQOL and IPVI) documentation of voice disorders. J Voice. 2007;21(5):576-90. http://dx.doi.org/10.1016/j.jvoice.2006.05.001. PMid: 16822648

14. Gasparini G, Behlau M. Quality of life: validation of the Brazilian version of the voice-related quality of life (V-RQOL) measure. J Voice. 2009;23(1):76-81. http://dx.doi.org/10.1016/j.jvoice.2007.04.005. PMid:17628396.

15. Moreti F, Zambon F, Oliveira G, Behlau M. Cross-cultural adaptation, validation, and cutoff values of the Brazilian version of the Voice Symptom Scale-VoiSS. J Voice. 2014;28(4):458-68. http://dx.doi. org/10.1016/j.jvoice.2013.11.009. PMid:24560004.

16. Teixeira LC, Rodrigues AL, Silva AF, Azevedo R, Gama AC, Behlau $M$. The use of the URICA-VOICE questionnaire to identify the stages of adherence to voice treatment. Codas. 2013;25(1):8-15. http://dx.doi. org/10.1590/S2317-17822013000100003. PMid:24408164.

17. Behlau M, Madazio G, Moreti F, Oliveira G, Santos LM, Paulinelli $\mathrm{BR}$, et al. Voice self-assessment protocols: different trends among organic and behavioral dysphonias. J Voice. 2017 Jan;31(1):112.e1327. http://dx.doi.org/10.1016/j.jvoice.2016.03.014. PMid:27210475.

18. Oliveira GG. Estratégias de enfrentamento nos distúrbios de voz [Tese]. São Paulo (SP): Universidade Federal de São Paulo; 2009.

19. Ribeiro MB, Gama ACC, Bassi IB, Teixeira LC. Parâmetros vocais, laríngeos e de autopercepção de professoras disfônicas: análise após tratamento fonoaudiologico. Rev CEFAC. 2013;15(3):616-41. http:// dx.doi.org/10.1590/S1516-18462013000300016.

20. Moreti F, Zambon F, Behlau M. Voice symptoms and vocal deviation self-assessment in different types of dysphonia. Codas. $2014 \mathrm{Jul}-$ Ago;26(4):331-3. http://dx.doi.org/10.1590/2317-1782/201420130036. PMid:25211694.

21. Likert RA. Technique for the measurement of attitudes. Arch Psychol. 1932;140:1-55

22. Castro SM, Trentini C, Riboldi J. Item response theory applied to the Beck Depression Inventory. Rev Bras Epidemiol. 2010 Set;13(3):487-501. http://dx.doi.org/10.1590/S1415-790X2010000300012. PMid:20857035.

23. Aaronson N, Alonso J, Burnam A, Lohr KN, Patrick DL, Perrin E, et al. Scientific Advisory Committee of the medical outcomes trust. Assessing health status and quality-of-life instruments: attributes and review criteria. Qual Life Res. 2002;11(3):193-205. http://dx.doi. org/10.1023/A:1015291021312. PMid:12074258.

24. Moher D, Liberati A, Tetzlaff J, Altman DG, PRISMA Group. Preferred reporting items for systematic reviews and meta-analyses: the PRISMA statement. Ann Intern Med. 2009;151(4):264-9, W64. http://dx.doi. org/10.7326/0003-4819-151-4-200908180-00135. PMid:19622511.

25. Paulinelli BR, Gama ACC, Behlau M. Validação do Questionário de Performance Vocal no Brasil. Rev Soc Bras Fonoaudiol. 2012;17(1):8591. http://dx.doi.org/10.1590/S1516-80342012000100016.

26. Ricarte A, Oliveira G, Behlau M. Validation of the Voice Activity and Participation Profile protocol in Brazil. Codas. 2013;25(3):2429. CoDAS. 2013;25(3):242-9. http://dx.doi.org/10.1590/S231717822013000300009. PMid:24408335. 
27. Oliveira G, Zambon F, Vaiano T, Costa F, Behlau M. Versões reduzidas para protocolo clínico de enfrentamento das disfonias. CoDAS 2016;28(6):828-32. http://dx.doi.org/10.1590/2317-1782/20162015177. PMid:27982248.

28. Krohling LL, Paula KM, Behlau M. ROC curve of the Pediatric Voice Related Quality-of-Life Survey (P-VRQOL). CoDAS. 2016 Maio 31;28(3):311-3. http://dx.doi.org/10.1590/2317-1782/20162015103. PMid:27253227.

29. Alexandre NMC, Coluci MZO. Validade de conteúdo nos processos de construção e adaptação de instrumentos de medidas. Ciênc. saúde coletiva. 2011;16(7):3061-8. http://dx.doi.org/10.1590/S141381232011000800006 .

30. Pernambuco L, Espelt A, Magalhães HV Jr, Lima KC. Recommendations for elaboration, transcultural adaptation and validation process of tests in Speech, Hearing and Language Pathology. CoDAS. 2017;29(3):e20160217. http://dx.doi.org/10.1590/2317-1782/20172016217. PMid:28614460.

31. Pasquali L. Psicometria. Rev Esc Enferm USP. 2009;43(Esp):992-9.

32. Raymundo VP. Construção e validação de instrumentos: um desafio para a psicolinguística. Let Hoje. 2009;44(3):86-93.

33. Bogaardt HC, Hakkesteegt MM, Grolman W, Lindeboom R. Validation of the voice handicap index using Rasch analysis. J Voice. 2007;21(3):337-47. http://dx.doi.org/10.1016/j.jvoice.2005.09.007. PMid:16504469.

34. Andrade DF, Tavares HR, Valle RC. Teoria de resposta ao item: conceitos e aplicações. São Paulo, SP: Associação Brasileira de Estatística; 2000.

35. Araujo EAC, Andrade DF, Bortolotti SLV. Teoria da Resposta ao Item. Rev Esc Enferm USP. 2009; 43(Esp):1000-8. http://dx.doi.org/10.1590/ S0080-62342009000500003.

36. Branski RC, Cukier-Blaj S, Pusic A, Cano SJ, Klassen A, Mener D, et al. Measuring quality of life in dysphonic patients: a systematic review of content development in patient-reported outcomes measures. J Voice. 2010;24(2):193-8. http://dx.doi.org/10.1016/j.jvoice.2008.05.006. PMid:19185454.

37. Pasquali L. Teoria de resposta ao item: teoria de procedimentos e aplicações. Brasília: LabPAM/UnB; 2007.

38. Seifpanahi S, Jalaie S, Nikoo MR, Sobhani-Rad D. Translated Versions of Voice Handicap Index (VHI)-30 across languages: a systematic review. Iran J Public Health. 2015;44(4):458-69. PMid:26056664.
39. Nawka T, Wiesmann U, Gonnermann U. Validierung des Voice Handicap Index (VHI) in der deutschen Fassung. HNO. 2003;51(11):921-30. http://dx.doi.org/10.1007/s00106-003-0909-8. PMid:14605713.

40. Wilson JA, Webb A, Carding PN, Steen IN, Mackenzie K, Deary IJ. The Voice Symptom Scale (VoiSS) and the Vocal Handicap Index (VHI): a comparison of structure and content. Clin Otolaryngol Allied Sci. 2004;29(2):169-74. http://dx.doi.org/10.1111/j.0307-7772.2004.00775.x. PMid:15113305.

41. Pasquali L. Análise fatorial para pesquisadores. Porto Alegre: Art Med.; 2005.

42. Yamasaki R, Madazio G, Leão SHS, Padovani M, Azevedo R, Behlau M. Auditory-perceptual evaluation of normal and dysphonic voices using the voice deviation scale. J Voice. 2017;31(1):67-71. http:// dx.doi.org/10.1016/j.jvoice.2016.01.004. PMid:26873420.

43. Mendonça KMPP, Guerra RO. Desenvolvimento e validação de um instrumento de medida da satisfação do paciente com a fisioterapia. Rev. Bras. Fisioter. 2007;11(5):369-76. http://dx.doi.org/10.1590/ S1413-35552007000500007.

44. Prochaska JO, DiClemente CC. Stages and processes of self-change of smoking: toward an integrative model of change. J Consult Clin Psychol. 1983;51(3):390-5. http://dx.doi.org/10.1037/0022-006X.51.3.390. PMid:6863699.

45. Fahning AKCA, Almeida AAF, Anjos UU. Terapia de grupo como facilitadora da adesão do paciente com disfonia comportamental [dissertação]. João Pessoa (PB). Universidade Federal da Paraíba; 2015.

46. Costa BOI, Silva POC, Pinheiro RSA, Silva HF, Almeida AAF. Stage of readiness of patients with behavioral dysphonia in pre and postgroup voice therapy assessments. CoDAS. 2017;29(4):e20160198. http://dx.doi.org/10.1590/2317-1782/20172016198. PMid:28813072.

47. Aguiar AC, Almeida LN, Pernambuco L, Palhano DB, Andrade JM, Behlau M, et al. Stages of readiness in patients with dysphonia: a proposal based on factor analysis using the URICA-V scale. J Voice. 2020;34(4):547-58. http://dx.doi.org/10.1016/j.jvoice.2018.12.004. PMid:30595237.

48. Rodrigues G, Zambon F, Mathieson L, Behlau M. Vocal tract discomfort in teachers: its relationship to self-reported voice disorders. J Voice. 2013;27(4):473-80. http://dx.doi.org/10.1016/j.jvoice.2013.01.005. PMid:23528674.

49. Santos HH, Aguiar AG, Baeck HE, Van Borsel J. Tradução e avaliação preliminar da versão em Português do Questionário de Autoavaliação Vocal para Transexuais de Homem para Mulher. CoDAS. 2015 JanFev;27(1):89-96. PMid:25885202. 